\title{
Approximate Controllability of Neutral Measure Evolution Equations with Nonlocal Conditions
}

\author{
Lina Ma, Haibo Gu $(\mathbb{D}$, and Yiru Chen \\ School of Mathematics Science, Xinjiang Normal University, Urumqi 830017, China \\ Correspondence should be addressed to Haibo Gu; hbgu_math@163.com
}

Received 6 October 2020; Revised 22 November 2020; Accepted 10 December 2020; Published 11 January 2021

Academic Editor: Muslim Malik

Copyright (C) 2021 Lina Ma et al. This is an open access article distributed under the Creative Commons Attribution License, which permits unrestricted use, distribution, and reproduction in any medium, provided the original work is properly cited.

In this paper, we consider a kind of neutral measure evolution equations with nonlocal conditions. By using semigroup theory and fixed point theorem, we can obtain sufficient conditions for the controllability results of such equations. Finally, an example is given to verify the reliability of the results.

\section{Introduction}

In the past decades, the theory of impulsive differential equations has been fully developed. An impulsive differential equation is used to simulate the evolution process of a system under perturbation during continuous evolution [1-3]. However, this type of system allows only a limited number of discontinuities within a limited range. As a result, it cannot simulate some complex phenomena, such as Zeno's behavior. However, the dynamic system with discontinuous trajectory is modeled by a measure differential equation or measure-driven equation [4-10]. Measure differential equations (MDEs) were studied in the early days [11-18]. In 1971, Das first studied measure differential equations. For a complete introduction of measure differential systems, we can refer to $[12,13]$.

On the other hand, the complete controllability of several nonlinear dynamic systems, such as stochastic systems of fractional order and dynamic systems of impulsive differential equations, has been extensively studied. Recently, some authors had discussed existence, stability, and nonlocal controllability of the measure evolution equation $[9,15,19-22]$. In the past few years, the existence and controllability of fractional abstract functional differential development systems with nonlocal conditions have been fully studied [2, 23-31]. However, the controllability problem of neutral measure evolution equations with nonlocal conditions is seldom studied. Therefore, whether the appropriate solution of the system exists for any given control $u$ and whether the system is approximately controllable are studied.

In the paper, we will study the following neutral measure evolution equations with nonlocal conditions:

$$
\left\{\begin{array}{l}
\mathrm{d}\left[x(t)-q\left(t, x_{t}\right)\right]=A x(t) \mathrm{d} t+\left[B u(t)+f\left(t, x_{t}\right)\right] \mathrm{d} g(t), \quad t \in J=[0, a], \\
x(s)=\phi(s)+p(x), \quad s \in[-r, 0],
\end{array}\right.
$$


where $f, q:[0, a] \times G([-r, 0] ; X) \longrightarrow X, x_{t}(\theta)=x(t+\theta)$, $\theta \in[-r, 0], r>0, \quad p: G([-r, a] ; X) \longrightarrow X$ is a specified function. The state variable $x(\cdot)$ takes values in Banach space $X$ with the norm $\|\cdot\| . A: D(A) \subset X \longrightarrow X$ generates a uniformly bounded analytic semigroup $\{T(t)\}_{t \geq 0}$ in Banach space $X . g:[0, a] \longrightarrow R$ is a nondecreasing leftcontinuous function. The control function $u(t)$ takes values in another Banach space $U$, where $U$ is a control set. The set $G([-r, 0] ; X)$ and $G([-r, a] ; X)$ represent the regulated functions space on $[-r, 0]$ and $[-r, a]$, respectively. The rest of this paper is organized as follows. In Section 2, some notations and preparation are given about Kurzweil-Henstock-Stieltjes integrals and regulated functions. In Section 3, we obtain the existence results for measure evolution system (1) by using Schauder's fixed point theorem. In Section 4, based on Krasnoselskii's fixed point theorem, we establish a controllability result for mild solutions of system (1). An example is given to prove validity of the results we obtained in Section 5.

\section{Preliminaries}

In this section, we will review some concepts and main results regarding Kurzweil-Henstock-Stieltjes integrals and regulated functions.

Consider a function $\delta:[a, b] \longrightarrow R^{+}$. A tagged partition of the interval $[a, b]$ with division points $a=t_{0} \leq t_{1} \leq \cdots \leq$ $t_{m}=b$ and tags $\tau_{i} \in\left[t_{i-1}, t_{i}\right], i=1, \ldots, m$ is called $\delta$-fine if $\tau_{i}-\delta\left(\tau_{i}\right) \leq t_{i} \leq \tau_{i}+\delta\left(\tau_{i}\right), i \in\{1, \ldots, m\}$.

Definition 1 (See [22]). Let $g:[a, b] \longrightarrow R$. A function $f:[a, b] \longrightarrow R^{n}$ is said to be Kurzweil-Henstock-Stieltjes integrable with respect to (w.r.t.) $g$ on $[a, b]$ (shortly, $\mathrm{K}-\mathrm{H}-$ Stieltjes integrable) if there exists a vector $I \in X$ such that for every $\varepsilon>0$, there is a gauge $\delta_{\varepsilon}$ on $[a, b]$ with

$$
\left\|\sum_{i=1}^{m} f\left(\tau_{i}\right)\left(g\left(t_{i}\right)-g\left(t_{i-1}\right)\right)-I\right\|<\varepsilon,
$$

for every $\delta_{\varepsilon}$-fine partition $\left\{\left(\left[t_{i-1}, t_{i}\right], \xi_{i}\right): i=1,2, \ldots, m\right\}$ of $[a, b]$. The $\mathrm{K}-\mathrm{H}-\mathrm{Stieltjes}$ integrability is preserved on all subintervals of $[a, b]$. The function $\int_{a}^{b} f(t) \mathrm{d} g(t)$ is called the $\mathrm{K}-\mathrm{H}-\mathrm{Stieltjes}$ primitive of $f$ w.r.t. $g$ on $[a, b]$. Let $K_{g}^{p}(J ; X)(p>1)$ be a space of all $p$-ordered $\mathrm{K}-\mathrm{H}$-Stieltjes integral regulated functions from $J$ to $X$ with respect to $g$, with the norm $\|\cdot\|_{K_{g}^{p}}$ defined by

$$
\|f\|_{K_{g}^{p}}=\left((\mathrm{KHS}) \int_{0}^{a}|f(s)|^{p} \mathrm{~d} g(s)\right)^{(1 / p)} .
$$

Definition 2 (See [7]). Let $X$ be a Banach space with a norm $\|\cdot\|$ and $[a, b]$ be a closed interval of the real line. A function $f:[a, b] \longrightarrow X$ is called regulated on $[a, b]$ if the limits

$$
\begin{array}{ll}
\lim _{s \longrightarrow t^{-}} f(s)=f\left(t^{-}\right), & t \in(a, b], \\
\lim _{s \longrightarrow t^{+}} f(s)=f\left(t^{+}\right), & t \in[a, b),
\end{array}
$$

exist and are finite. The space of all regulated functions $f:[a, b] \longrightarrow X$ is denoted by $G([a, b] ; X)$. It is well known that the set of discontinuities of a regulated function is at most countable and that the space $G([a, b] ; X)$ is a Banach space endowed with the norm $\|f\|_{\infty}=\sup _{t \in[a, b]}|f(t)|$. Let $r>0$, for any element $z \in G([-r, 0] ; X)$, and we define the norm $\|z\|_{*}=\sup _{s \in[-r, 0]}|z(s)|$.

Let $Y$ be another separable reflexive Banach space where control function $u$ takes values. Let $E \subset Y$ be bounded, and admissible control set $U=K_{g}^{p}(J ; E)(p>1)$.

Definition 3 (See [7]). A set $\mathscr{A} \subset G([a, b] ; X)$ is called equiregulated if for every $\varepsilon>0$ and $t_{0} \in[a, b]$, there is a $\theta>0$ such that

(i) If $x \in \mathscr{A}, t \in[a, b]$ and $t_{0}-\theta<t<t_{0}$, then $\left|x\left(t_{0}^{-}\right)-x(t)\right|<\varepsilon$

(ii) If $x \in \mathscr{A}, t \in[a, b]$ and $t_{0}<t<t_{0}+\theta$, then $\left|x(t)-x\left(t_{0}^{+}\right)\right|<\varepsilon$

Lemma 1 (See [7]). Consider the functions $f: J \longrightarrow X$ and $g: J \longrightarrow R$ such that $g$ is regulated and $\int_{a}^{b} f d g$ exists. Then, the function $h(t)=\int_{a}^{b} f d g, t \in[a, b]$ is regulated and satisfies

$$
\begin{array}{ll}
h\left(t^{+}\right)=h(t)+f(t) \Delta^{+} g(t), & t \in[a, b), \\
h\left(t^{-}\right)=h(t)-f(t) \Delta^{-} g(t), & t \in(a, b],
\end{array}
$$

where $\Delta^{+} g(t)=g\left(t^{+}\right)-g(t)$ and $\Delta^{-} g(t)=g(t)-g\left(t^{-}\right)$, where $g\left(t^{-}\right)$and $g\left(t^{+}\right)$denote the left limit and the right limit of the function $g$ at $t$, respectively.

Lemma 2 (See [7]). Let $X$ be a Banach space. Assume that $\mathscr{A} \subset G([a, b] ; X)$ is equiregulated, and for every $t \in[a, b]$, the set $\{x(t): t \in \mathscr{A}\}$ is relatively compact in $X$. Then, the set $\mathscr{A}$ is relatively compact in $G([a, b] ; X)$.

Lemma 3 (See [7]). Let $X$ be a Banach space and $E \subseteq X$ be a bounded, closed, and convex set. If the operator $N: E \longrightarrow E$ is completely continuous, then $N$ has a fixed point on $E$.

Lemma 4 (See [7]). Let $\left\{x_{n}\right\}_{n=1}^{\infty}$ be a sequence of functions from $[a, b]$ to $X$. If $x_{n}$ converges pointwisely to $x_{0}$ as $n \longrightarrow \infty$ and the sequence $\left\{x_{n}\right\}_{n=1}^{\infty}$ is equiregulated, then $x_{n}$ converges uniformly to $x_{0}$.

For $0<\alpha<1,(-A)^{\alpha}$ can be defined as a closed linear invertible operator with its domain $D(-A)^{\alpha}$ being dense in $X$. We denote by $X_{\alpha}$ the Banach space $D(-A)^{\alpha}$ endowed with norm $\|x\|_{\alpha}=\left\|(-A)^{\alpha} x\right\|$ which is equivalent to the graph 
norm of $(-A)^{\alpha}$. We have $X_{\beta} \hookrightarrow X_{\alpha}$ for $0<\alpha<\beta$, and the embedding is continuous [32].

Lemma 5 (See [32]). ie following properties hold:

(1) If $0<\alpha<\beta$, then $X_{\alpha} \subset X_{\beta}$ and the embedding is compact whenever the resolvent operator of $A$ is compact

(2) For every $0<\alpha<1$, there exists $C_{\alpha}>0$ such that $\left\|(-A)^{\alpha} T(t)\right\| \leq\left(C_{\alpha} / t^{\alpha}\right)$

Lemma 6 (See [31]). Let $M$ be a closed convex nonempty subset of a Banach space $(S,|\cdot|)$. We suppose that $P$ and $Q$ map $M$ into $S$ such that

(i) $P x+Q y \in M(\forall x, y \in M)$

(ii) $P$ is continuous and $P M$ is contained in a compact set

(iii) $Q$ is a contraction with constant $k<1$

Then, there is a $y \in M$ with $P y+Q y=y$.

\section{Existence Results}

In this section, we will study the existence of the mild solution of system (1). We first give the definition of solutions for measure system (1). Using the methods same as in [33], we can obtain the following definition.

Definition 4. The function $x \in G(J ; X)$ is called a mild solution of system (1) on $J$ if it satisfies the following measure integral equation:

$$
\begin{aligned}
x(t)= & T(t)\left[\phi(0)+p(x)-q\left(0, x_{0}\right)\right]+q\left(t, x_{t}\right) \\
& +\int_{0}^{t} A T(t-s) q\left(s, x_{s}\right) \mathrm{d} s \\
& +\int_{0}^{t} T(t-s)\left[B u(s)+f\left(s, x_{s}\right)\right] \mathrm{d} g(s) .
\end{aligned}
$$

We introduce the following assumptions:

(H1): let $M=\sup _{t \in[0, a]}|T(t)|$, and $T(t)$ is a compact operator for every $t>0$

$(\mathrm{H} 2)$ : for each $t \in[0, a]$, the function $q$ : $[0, a] \times$ $G([-r, 0] ; X) \longrightarrow X$ is continuous and there exists a constant $\alpha \in(0,1)$, and $H, H_{1}>0$ such that $q \in D\left((-A)^{\alpha}\right)$, and for any $\phi_{1}, \phi_{2}, \phi \in G([-r, 0]$; $X), t \in[0, a]$, the function $(-A)^{\alpha} q(\cdot, \phi)$ is strongly measurable and $(-A)^{\alpha} q(t, \cdot)$ satisfies the Lipschitz condition,

$$
\left|(-A)^{\alpha} q\left(t, \phi_{1}\right)-(-A)^{\alpha} q\left(t, \phi_{2}\right)\right| \leq H\left\|\phi_{1}-\phi_{2}\right\|_{*},
$$

and the inequality

$$
\left|(-A)^{\alpha} q(t, \phi)\right| \leq H_{1}\left(\|\phi\|_{*}+1\right)
$$

(H3): the function $f:[0, a] \times G([-r, 0] ; X) \longrightarrow X$ satisfies the following:

(i) $f(\cdot, \phi)$ is measurable for all $\phi \in G([-r, 0] ; X)$, and $f(t, \cdot): G([-r, 0] ; X) \longrightarrow K_{g}^{p}(J ; X)$ is continuous for a.e.t $\in J$

(ii) There is a function $m \in K_{g}^{p}(J ; X)$ and a nondecreasing continuous function $\Phi: R^{+} \longrightarrow R^{+}$such that

$$
\left|f\left(t, x_{t}\right)\right| \leq m(t) \Phi(\|x\|),
$$

for all $x_{t} \in G([-r, 0] ; X)$, almost all $t \in J$, and $\lim _{l \longrightarrow+\infty} \inf (\Phi(l) / l)=\varphi<+\infty$

(H4): $p: G([-r, a] ; X) \longrightarrow X$ is continuous and compact, and there exist positive constants $e$ and $f$ such that

$$
\|p(x)\| \leq e\|x\|_{\infty}+f,
$$

(H5): $B: E \longrightarrow X$ is a linear and bounded operator, so there is a positive constant $M_{B}$ such that $\|B\| \leq M_{B}$

Theorem 1. If assumptions (H1)-(H5) hold, then problem (1) has a mild solution provided that

$$
\begin{gathered}
M e+(M+1)\left\|(-A)^{-\alpha}\right\| H_{1}+\frac{C_{1-\alpha} H_{1} a^{\alpha}}{\alpha} \\
+M \varphi[g(a)-g(0)]^{(1 / q)}\|m\|_{K_{g}^{p}<1 .}
\end{gathered}
$$

Proof. We define the operator Q: $G(J ; X) \longrightarrow G(J ; X)$ by

$$
\begin{aligned}
(Q x)(t)= & T(t)\left[\phi(0)+p(x)-q\left(0, x_{0}\right)\right]+q\left(t, x_{t}\right) \\
& +\int_{0}^{t} A T(t-s) q\left(s, x_{s}\right) \mathrm{d} s \\
& +\int_{0}^{t} T(t-s)\left[B u(s)+f\left(s, x_{s}\right)\right] \mathrm{d} g(s) .
\end{aligned}
$$

Let $l>0$ be a constant and $B_{l}=\left\{G(J ; X):\|x\|_{\infty} \leq l\right\}$, where $B_{l}$ is a bounded, closed, and convex set, $Q\left(B_{l}\right)=\left\{Q(x): x \in B_{l}\right\}$.

Step I: we prove that there exists a constant $l>0$ such that $Q\left(B_{l}\right) \subset B_{l}$. Assuming that this conclusion is not true, for each $l>0$, there will exist $x^{l} \in B_{l}, t_{l} \in J$ such that $\left\|Q x^{l}\left(t_{l}\right)\right\|>l$.

According to (H1)-(H5), 


$$
\begin{aligned}
& \left\|Q x^{l}\left(t_{l}\right)\right\| \\
& =\left\|T\left(t_{l}\right)\left[\phi(0)+p\left(x^{l}\right)-q\left(0, x_{0}\right)\right]+q\left(t_{l}, x_{t_{l}}^{l}\right)+\int_{0}^{t_{l}} A T\left(t_{l}-s\right) q\left(s, x_{s}^{l}\right) \mathrm{d} s+\int_{0}^{t_{l}} T\left(t_{l}-s\right)\left[B u(s)+f\left(s, x_{s}^{l}\right)\right] \mathrm{d} g(s)\right\| \\
& \leq M\left\|\phi(0)+p\left(x^{l}\right)\right\|+M\left\|q\left(0, x_{0}\right)\right\|+\left\|q\left(t_{l}, x_{t_{l}}^{l}\right)\right\|+\left\|\int_{0}^{t_{l}}(-A)^{1-\alpha} T\left(t_{l}-s\right)(-A)^{\alpha} q\left(s, x_{s}^{l}\right) \mathrm{d} s\right\| \\
& +\left\|\int_{0}^{t_{l}} T\left(t_{l}-s\right) B u(s) \mathrm{d} g(s)\right\|+\left\|\int_{0}^{t_{l}} T\left(t_{l}-s\right) f\left(s, x_{s}^{l}\right) \mathrm{d} g(s)\right\| \\
& \leq M\|\phi(0)+e\| x^{l}\|+f\|+M\left\|(-A)^{-\alpha}\right\| H_{1}\left(\left\|x_{0}\right\|+1\right)+\left\|(-A)^{-\alpha}\right\| H_{1}\left(\left\|x^{l}\right\|+1\right)+C_{1-\alpha} H_{1} \\
& \times\left(\left\|x^{l}\right\|+1\right)\left\|\int_{0}^{t_{l}}\left(t_{l}-s\right)^{\alpha-1} \mathrm{~d} s\right\|+M M_{B}\left\|\int_{0}^{t_{l}} u(s) \mathrm{d} g(s)\right\|+M\left\|\int_{0}^{t_{l}} f\left(s, x_{s}^{l}\right) \mathrm{d} g(s)\right\| \\
& \leq M\|\phi(0)+e l+f\|+(M+1)\left\|(-A)^{-\alpha}\right\| H_{1}(l+1)+\frac{C_{1-\alpha} H_{1}(l+1) a^{\alpha}}{\alpha} \\
& +M M_{B}[g(a)-g(0)]^{(1 / q)}\|u\|_{K_{g}^{p}}+M[g(a)-g(0)]^{(1 / q)} \Phi\left(\left\|x^{l}\right\|\right)\left(\int_{0}^{t_{l}}|m(s)|^{p} \mathrm{~d} g(s)\right)^{(1 / p)} \\
& \leq M\|\phi(0)+e l+f\|+(M+1)\left\|(-A)^{-\alpha}\right\| H_{1}(l+1)+\frac{C_{1-\alpha} H_{1}(l+1) a^{\alpha}}{\alpha} \\
& +M M_{B}[g(a)-g(0)]^{(1 / q)}\|u\|_{K_{g}^{p}}+M[g(a)-g(0)]^{(1 / q)} \Phi(l)\|m\|_{K_{g}^{p} .}
\end{aligned}
$$

In the formula (13), $q>0,(1 / q)+(1 / p)=1$, combining

(13) with the fact $l<\left\|Q x^{l}\left(t_{l}\right)\right\|$, we can obtain

$$
\begin{aligned}
l<\left\|Q x^{l}\left(t_{l}\right)\right\| \leq & M\|\phi(0)+e l+f\|+(M+1)\left\|(-A)^{-\alpha}\right\| H_{1}(l+1)+\frac{C_{1-\alpha} H_{1}(l+1) a^{\alpha}}{\alpha} \\
& +M M_{B}[g(a)-g(0)]^{(1 / q)}\|u\|_{K_{g}^{p}}+M[g(a)-g(0)]^{(1 / q)} \Phi(l)\|m\|_{K_{g}^{p} .}
\end{aligned}
$$

Dividing both sides of (14) by $l$ and taking limit as

$l \longrightarrow \infty$, we have

$$
M e+(M+1)\left\|(-A)^{-\alpha}\right\| H_{1}+\frac{C_{1-\alpha} H_{1} a^{\alpha}}{\alpha}+M \varphi[g(a)-g(0)]^{(1 / q)}\|m\|_{K_{g}^{p}}>1,
$$

which contradicts.

Step II: $Q\left(B_{l}\right)$ is equiregulated. 


$$
\begin{aligned}
\left\|Q x(t)-Q x\left(t_{0}^{+}\right)\right\| \leq & \left\|T(t)-T\left(t_{0}^{+}\right)\right\|\left\|\phi(0)+p(x)-q\left(0, x_{0}\right)\right\|+\left\|q\left(t, x_{t}\right)-q\left(t_{0}^{+}, x_{t_{0}^{+}}\right)\right\| \\
& +\left\|\int_{0}^{t_{0}^{+}}(-A)\left[T(t-s)-T\left(t_{0}^{+}-s\right)\right] q\left(s, x_{s}\right) \mathrm{d} s\right\|+\left\|\int_{t_{0}^{+}}^{t}(-A) T(t-s) q\left(s, x_{s}\right) \mathrm{d} s\right\| \\
& +\left\|\int_{0}^{t_{0}^{+}}\left[T(t-s)-T\left(t_{0}^{+}-s\right)\right] B u(s) \mathrm{d} g(s)\right\|+\left\|\int_{t_{0}^{+}}^{t} T(t-s) B u(s) \mathrm{d} g(s)\right\| \\
& +\left\|\int_{0}^{t_{0}^{+}}\left[T(t-s)-T\left(t_{0}^{+}-s\right)\right] f\left(s, x_{s}\right) \mathrm{d} g(s)\right\|+\left\|\int_{t_{0}^{+}}^{t} T(t-s) f\left(s, x_{s}\right) \mathrm{d} g(s)\right\| \\
= & I_{1}+I_{2}+I_{3}+I_{4}+I_{5}+I_{6}+I_{7}+I_{8},
\end{aligned}
$$

where

$$
\begin{aligned}
& I_{1}=\left\|T(t)-T\left(t_{0}^{+}\right)\right\|\left\|\phi(0)+p(x)-q\left(0, x_{0}\right)\right\|, \\
& I_{2}=\left\|q\left(t, x_{t}\right)-q\left(t_{0}^{+}, x_{t_{0}^{+}}\right)\right\|, \\
& I_{3}=\left\|\int_{0}^{t_{0}^{+}}(-A)\left[T(t-s)-T\left(t_{0}^{+}-s\right)\right] q\left(s, x_{s}\right) \mathrm{d} s\right\|, \\
& I_{4}=\left\|\int_{t_{0}^{+}}^{t}(-A) T(t-s) q\left(s, x_{s}\right) \mathrm{d} s\right\|, \\
& I_{5}=\left\|\int_{0}^{t_{0}^{+}}\left[T(t-s)-T\left(t_{0}^{+}-s\right)\right] B u(s) \mathrm{d} g(s)\right\|, \\
& I_{6}=\left\|\int_{t_{0}^{+}}^{t} T(t-s) B u(s) \mathrm{d} g(s)\right\|, \\
& I_{7}=\left\|\int_{0}^{t_{0}^{+}}\left[T(t-s)-T\left(t_{0}^{+}-s\right)\right] f\left(s, x_{s}\right) \mathrm{d} g(s)\right\|, \\
& I_{8}=\left\|\int_{t_{0}^{+}}^{t} T(t-s) f\left(s, x_{s}\right) \mathrm{d} g(s)\right\| .
\end{aligned}
$$

The combination of the compactness of semigroup $T(t),(t>0)$ and its strong continuity shows the continuity of $T(t)$ in the sense of uniform operator topology and $p(x), q\left(t, x_{t}\right), f\left(t, x_{t}\right)$ are bounded, and applying dominated convergence theorem, $I_{1}, I_{3}$, $I_{5}, I_{7} \longrightarrow 0$, as $t \longrightarrow t_{0}^{+}$, independently on particular choices of $x(\cdot)$. Combining $1=(-A)^{-\alpha}(-A)^{\alpha}$ and $(-A)=(-A)^{\alpha}(-A)^{1-\alpha}$ with $(\mathrm{H} 2), I_{2}, I_{4} \longrightarrow 0$.

$$
\begin{aligned}
I_{6} & =\left\|\int_{t_{0}^{+}}^{t} T(t-s) B u(s) \mathrm{d} g(s)\right\| \\
& \leq M M_{B}\|u\|_{K_{g}^{p}}\left[g(t)-g\left(t_{0}^{+}\right)\right]^{(1 / q)},
\end{aligned}
$$

as $t \longrightarrow t_{0}^{+}, I_{6} \longrightarrow 0$. Moreover, let $H(t)=$ $\int_{0}^{t} m(s) \mathrm{d} g(s)$, according to Lemma 1 , and $H(t)$ is a regulated function; then,

$$
I_{8} \leq\left\|\int_{t_{0}^{+}}^{t} T(t-s) f\left(s, x_{s}\right) \mathrm{d} g(s)\right\| \leq M \Phi(l)\left\|\int_{t_{0}^{+}}^{t} m(s) \mathrm{d} g(s)\right\| \leq M \Phi(l)\left\|H(t)-H\left(t_{0}^{+}\right)\right\| \longrightarrow 0,
$$

as $t \longrightarrow t_{0}^{+}$. Also, we can follow the similar procedure to show $\left\|(Q x)\left(t_{0}^{-}\right)-(Q x)(t)\right\| \longrightarrow 0$ as $t \longrightarrow t_{0}^{-}$for each $t_{0} \in(0, a]$. Therefore, $Q\left(B_{l}\right)$ is equiregulated on $J$.

Step III: $Q: B_{l} \longrightarrow B_{l}$ is a continuous operator. Let $x^{n}$ be a convergent sequence in $B_{l}$ with $x^{n} \longrightarrow x \in B_{l}$ as $n \longrightarrow \infty$. According to hypothesis (H2)-(H5) and the boundedness of $T(t)$, we have, for each $s \in J$,

$$
\begin{gathered}
f\left(s, x_{s}^{n}\right) \longrightarrow f\left(s, x_{s}\right), q\left(s, x_{s}^{n}\right) \longrightarrow q\left(s, x_{s}\right), \\
\cdot p\left(x^{n}\right) \longrightarrow p(x),
\end{gathered}
$$


as $n \longrightarrow \infty$, and then, by the dominated convergence

theorem, we get

$$
\begin{aligned}
\left\|Q\left(x^{n}\right)-Q(x)\right\| \leq & M\left\|p\left(x^{n}\right)-p(x)\right\|+\left\|q\left(t, x_{t}^{n}\right)-q\left(t, x_{t}\right)\right\| \\
& +\left\|\int_{0}^{t}(-A)^{1-\alpha} T(t-s)(-A)^{\alpha}\left[q\left(s, x_{s}^{n}\right)-q\left(s, x_{s}\right)\right] \mathrm{d} s\right\| \\
& +\int_{0}^{t} T(t-s)\left\|f\left(s, x_{s}^{n}\right)-f\left(s, x_{s}\right)\right\| \mathrm{d} g(s) \\
\leq & M\left\|p\left(x^{n}\right)-p(x)\right\|+\left\|q\left(t, x_{t}^{n}\right)-q\left(t, x_{t}\right)\right\| \\
& +C_{1-\alpha} \int_{0}^{t}(t-s)^{\alpha-1}(-A)^{\alpha}\left\|q\left(s, x_{s}^{n}\right)-q\left(s, x_{s}\right)\right\| \mathrm{d} s \\
& +M \int_{0}^{t}\left\|f\left(s, x_{s}^{n}\right)-f\left(s, x_{s}\right)\right\| \mathrm{d} g(s) \longrightarrow 0 .
\end{aligned}
$$

In addition, the analysis same as in Step II demonstrates that $\left\{Q x^{n}\right\}_{n=1}^{\infty}$ is equiregulated on $J$. This property and the abovementioned verification together with Lemma 4 imply that $Q x^{n}$ converges uniformly to $Q x(t)$ as $n \longrightarrow \infty$, namely,

$$
\begin{array}{r}
\left\|Q x^{n}-Q x\right\|_{\infty}=\sup _{t \in J}\left|Q x^{n}(t)-Q x(t)\right| \longrightarrow 0, \\
\text { as } n \longrightarrow \infty .
\end{array}
$$

Therefore, $Q B_{l}$ is a continuous operator on $J$.

Step IV: for every $t \in J$, the set $V(t)=\left\{(Q x)(t): x(\cdot) \in B_{l}\right\}$ is relatively compact in $X$.

The case where $t=0$ is trivial, since $V(0)=\left\{\phi(0)+p(x), x \in B_{l}\right\}$. Let $t(0 \leq t \leq a)$ be fixed, and let $\eta$ be a given real number satisfying $0<\eta<t$. For every $x(\cdot) \in B_{l}$, we define

$$
\begin{aligned}
\left(Q_{\eta} x\right)(t)= & T(t)\left[\phi(0)+p(x)-q\left(0, x_{0}\right)\right]+q\left(t, x_{t}\right)+T(\eta) \int_{0}^{t-\eta} A T(t-s-\eta) q\left(s, x_{s}\right) \mathrm{d} s \\
& +T(\eta) \int_{0}^{t-\eta} T(t-s-\eta)\left[B u(s)+f\left(s, x_{s}\right)\right] \mathrm{d} g(s) .
\end{aligned}
$$

Since $T(\eta)$ is compact, the set $V_{\eta}(t)=\left\{\left(Q_{\eta} x\right)(t)\right.$ : $x(\cdot) \in B_{l}$ \} is relatively compact in $X$ for every $\eta, 0<\eta<t$. On the other hand, for every $x(\cdot) \in B_{l}$ in view of assumption (H2)-(H3), we have

$$
\begin{aligned}
\left\|(Q x)-\left(Q_{\eta} x\right)\right\|= & \left\|\int_{t-\eta}^{t}(-A)^{1-\alpha} T(t-s)(-A)^{\alpha} q\left(s, x_{s}\right) \mathrm{d} s\right\|+\left\|\int_{t-\eta}^{t} T(t-s) B u(s) \mathrm{d} g(s)\right\| \\
& +\left\|\int_{t-\eta}^{t} T(t-s) f\left(s, x_{s}\right) \mathrm{d} g(s)\right\| \\
\leq & C_{1-\alpha} H_{1}(l+1)\left\|\int_{t-\eta}^{t}(t-s)^{\alpha-1} \mathrm{~d} s\right\|+M M_{B}\left\|\int_{t-\eta}^{t} u(s) \mathrm{d} g(s)\right\| \\
& +M \int_{t-\eta}^{t}\left\|f\left(s, x_{s}\right)\right\| \mathrm{d} g(s) \\
\leq & \frac{C_{1-\alpha} H_{1}(l+1) \eta^{\alpha}}{\alpha}+M M_{B}[g(a)-g(a-\eta)]^{(1 / q)}\|u\|_{K_{g}^{p}} \\
& +M \Phi(l)\left\|\int_{0}^{t} m(s) \mathrm{d} g(s)-\int_{0}^{t-\eta} m(s) \mathrm{d} g(s)\right\| \longrightarrow 0 .
\end{aligned}
$$

By the left continuity of $g$ and Lemma 1 , as $\eta \longrightarrow 0^{+}$, $\left\|(Q x)-\left(Q_{\eta} x\right)\right\| \longrightarrow 0$. Therefore there are relatively compact sets arbitrarily close to the set $V(t)$. Hence, for each $t \in J, V(t)$ is a relatively compact set in $X$. Step II and Step IV together with Lemma 2 imply that the set $Q\left(B_{l}\right)$ is relatively compact in $G(J ; X)$. Hence, $Q$ is a completely continuous operator on $B_{l}$. By Schauder's fixed point theorem (Lemma 3), $Q$ has a fixed point in $B_{l}$, which is a mild solution of measure control system (1). The proof is completed. 


\section{Controllability Result}

In this section, to investigate the controllability of system (1), we first give the definition of controllability.

Definition 5. The system (1) is said to be controllable on the interval $J$ if for every initial function $x_{0} \in G([-r, 0] ; X)$ and $x_{1} \in X$, there exists a control $u \in K_{g}^{p}(J ; E)(p>1)$ such that the mild solution $x(t)$ of (1) satisfies $x(a)=x_{1}$.

We assume the following conditions:

(H6): for all $\phi_{1}, \phi_{2} \in G([-r, 0] ; X)$, we assume that function $f$ satisfies

$$
\left|f\left(s, \phi_{1}\right)-f\left(s, \phi_{2}\right)\right| \leq K_{1}\left\|\phi_{1}-\phi_{2}\right\|_{*,}
$$

and for all $\varphi_{1}, \varphi_{2} \in G([-r, a] ; X)$, function $p$ satisfies

$$
\left|p\left(\varphi_{1}\right)-p\left(\varphi_{2}\right)\right| \leq L_{p}\left\|\varphi_{1}-\varphi_{2}\right\| \text {. }
$$

(H7): the linear operator $W: K_{g}^{p}(J ; U) \longrightarrow X$, defined by $W u=\int_{0}^{a} T(a-s) B u(s) \mathrm{d} g(s)$, has an invertible operator $W^{-1}$ taking values in $K_{g}^{p}(J ; U) / \operatorname{Ker} W$, and there exists a positive constant $M^{\prime}$ such that $\left\|B W^{-1}\right\| \leq M^{\prime}$

(H8): $\quad N=M \Phi\left(l^{\prime}\right)[g(a)-g(0)]^{(1 / q)}\|m\|_{K_{g}^{p}}+M M^{\prime}$ $[g(a)-g(0)]\left\{\left\|x_{1}\right\|+M\left[\|\phi(0)\|+e l^{\prime}+f+H_{1}\right.\right.$ $\left.\left\|(-A)^{-\alpha}\right\| \times\left(\left\|x_{0}\right\|+1\right)\right]+\left\|(-A)^{-\alpha}\right\| H_{1}\left(l^{\prime}+1\right)+$ $\left(\left(C_{1-\alpha} a^{\alpha} H_{1}\left(l^{\prime}+1\right)\right) / \alpha\right)+M \Phi\left(l^{\prime}\right)[g(a)-g(0)]^{(1 / q)}$ $\left.\|m\|_{K_{g}^{p}}\right\} \quad+M\left[e l^{\prime}+f+H_{1}\left\|(-A)^{-\alpha}\right\| \quad\left(\left\|x_{0}\right\|+1\right)\right]+H_{1}$ $\left\|(-A)^{-\alpha}\right\|\left(l^{\prime}+1\right)+\left(\left(C_{1-\alpha} a^{\alpha} H_{1}\left(l^{\prime}+1\right)\right) / \alpha\right)<l$,

where $l^{\prime}=l+M|\phi(0)|$

(H9): $k:=M L_{p}+H\left(\left\|(-A)^{-\alpha}\right\|+\left(a^{\alpha} C_{1-\alpha} / \alpha\right)\right)<1$

Theorem 2. If hypotheses (H1)-(H9) are satisfied, system (1) is controllable on $\mathrm{J}$.

Proof. Using assumption (H7) for an arbitrary function $x(\cdot)$, we define the control

$$
\begin{aligned}
u_{x}(t)= & W^{-1}\left[x_{1}-T(a)\left[\phi(0)+p(x)-q\left(0, x_{0}\right)\right]-q\left(a, x_{a}\right)-\int_{0}^{a} A T(a-s) q\left(s, x_{s}\right) \mathrm{d} s\right. \\
& \left.-\int_{0}^{a} T(a-s) f\left(s, x_{s}\right) \mathrm{d} g(s)\right](t) .
\end{aligned}
$$

In what follows, it suffices to show that when using this control, the operator $F$ defined by

$$
\begin{aligned}
F x(t)= & \phi(t)+p(x), t \in[-r, 0] \\
F x(t)= & T(t)\left[\phi(0)+p(x)-q\left(0, x_{0}\right)\right]+q\left(t, x_{t}\right)+\int_{0}^{t} A T(t-s) q\left(s, x_{s}\right) \mathrm{d} s \\
& +\int_{0}^{t} T(t-s)\left[B u_{x}(s)+f\left(s, x_{s}\right)\right] \mathrm{d} g(s), \quad t \in J,
\end{aligned}
$$

has a fixed point $x(\cdot)$ from which it follows that this fixed point is a mild solution of system (1). Clearly, $x(a)=(F x)(a)=x_{1}$, from which we conclude that the system is controllable. Let $x(t)=y(t)+\widehat{\phi}(t), t \in[-r, a]$, where $\widehat{\phi}(t)$ is taken as $\phi(t)+p(x)$ for $t \in[-r, 0]$ while for $t \in J$, it is defined as $T(t) \phi(0)$.

We define the operators $F_{1}$ and $F_{2}$ by

$$
\begin{aligned}
& F_{1} y(t)=\left\{\begin{array}{l}
0, \quad t \in[-r, 0], \\
\int_{0}^{t} T(t-s) f\left(s, y_{s}+\widehat{\phi}_{s}\right) \mathrm{d} g(s)+\int_{0}^{t} T(t-s) B W^{-1}\left[x_{1}-T(a)[\phi(0)+p(y+\widehat{\phi})\right. \\
\left.-q\left(0, x_{0}\right)\right]-q\left(a, y_{a}+\widehat{\phi}_{a}\right)-\int_{0}^{a} A T(a-\tau) q\left(\tau, y_{\tau}+\widehat{\phi}_{\tau}\right) \mathrm{d} \tau \\
\left.-\int_{0}^{a} T(a-s) f\left(\tau, y_{\tau}+\widehat{\phi}_{\tau}\right) \mathrm{d} g(\tau)\right](s) \mathrm{d} g(s), \quad t \in J,
\end{array}\right. \\
& F_{2} y(t)=\left\{\begin{array}{l}
0, t \in[-r, 0], \\
T(t)\left[p(y+\widehat{\phi})-q\left(0, x_{0}\right)\right]+q\left(t, y_{t}+\widehat{\phi}_{t}\right)+\int_{0}^{t} A T(t-s) q\left(s, y_{s}+\widehat{\phi}_{s}\right) \mathrm{d} s, \quad t \in J .
\end{array}\right.
\end{aligned}
$$


Obviously, the operator $F$ has a fixed point if only if operator $F_{1}+F_{2}$ has a fixed point. Thus, we shall employ Krasnoselskii's fixed point theorem.
Step I: we have to show $\left(F_{1}+F_{2}\right) B_{l} \subset B_{l}$, i.e., any $\phi_{1}, \phi_{2} \in B_{l}$ implies that $F_{1} \phi_{1}+F_{2} \phi_{2} \in B_{l}$. It is easy from hypotheses $(\mathrm{H} 1-\mathrm{H} 9)$ and Lemma 1 to see that

$$
\begin{aligned}
& \left\|F_{1} \phi_{1}(t)+F_{2} \phi_{2}(t)\right\|=\left\|\int_{0}^{t} T(t-s) f\left(s, \phi_{1 s}+\widehat{\phi}_{s}\right) \mathrm{d} g(s)\right\| \\
& +\int_{0}^{t} T(t-s) B W^{-1}\left[\left\|x_{1}\right\|+\left\|T(a)\left[\phi(0)+p\left(\phi_{1}+\widehat{\phi}\right)+q\left(0, x_{0}\right)\right]\right\|\right. \\
& +\left\|q\left(a, \phi_{1 a}+\widehat{\phi}_{a}\right)\right\|+\left\|\int_{0}^{a} A T(a-\tau) q\left(\tau, \phi_{1 \tau}+\widehat{\phi}_{\tau}\right) \mathrm{d} \tau\right\| \\
& \left.+\left\|\int_{0}^{a} T(a-s) f\left(\tau, \phi_{1 \tau}+\widehat{\phi}_{\tau}\right) \mathrm{d} g(\tau)\right\|\right](s) \mathrm{d} g(s) \\
& +\left\|T(t)\left[p\left(\phi_{2}+\widehat{\phi}\right)+q\left(0, x_{0}\right)\right]\right\|+\left\|q\left(t, \phi_{2 t}+\widehat{\phi}_{t}\right)\right\| \\
& +\left\|\int_{0}^{t} A T(t-s) q\left(s, \phi_{2 s}+\widehat{\phi}_{s}\right) \mathrm{d} s\right\| \\
& \leq M \Phi\left(l^{\prime}\right)[g(a) t-n g q(0)]^{(1 / q)}\|m\|_{K_{g}^{p}}+M M^{\prime}[g(a)-g(0)]\left\{\left\|x_{1}\right\|+M\left[\|\phi(0)\|+e l^{\prime}\right.\right. \\
& \left.+f+H_{1}\left\|(-A)^{-\alpha}\right\|\left(\left\|x_{0}\right\|+1\right)\right]+\left\|(-A)^{-\alpha}\right\| H_{1}\left(l^{\prime}+1\right)+\frac{C_{1-\alpha} a^{\alpha} H_{1}\left(l^{\prime}+1\right)}{\alpha} \\
& \left.+M \Phi\left(l^{\prime}\right)[g(a)-g(0)]^{(1 / q)}\|m\|_{K_{g}^{p}}\right\}+M\left[e l^{\prime}+f+H_{1}\left\|(-A)^{-\alpha}\right\|\left(\left\|x_{0}\right\|+1\right)\right] \\
& +H_{1}\left\|(-A)^{-\alpha}\right\|\left(l^{\prime}+1\right)+\frac{C_{1-\alpha} a^{\alpha} H_{1}\left(l^{\prime}+1\right)}{\alpha}=N<l,
\end{aligned}
$$

and thus, condition (i) in Lemma 6 is verified.

Step II: next, we need to show that operator $F_{1}$ is continuous.

$$
\begin{aligned}
\left|F_{1} \phi_{1}(t)-F_{1} \phi_{2}(t)\right|=\mid & \int_{0}^{t} T(t-s)\left[f\left(s, \phi_{1 s}+\widehat{\phi}_{s}\right)-f\left(s, \phi_{2 s}+\widehat{\phi}_{s}\right)\right] \mathrm{d} g(s) \mid+\int_{0}^{t} T(t-s) B W^{-1} \\
& \cdot\left[T(t)\left|p\left(\phi_{1 s}+\widehat{\phi}_{s}\right)-p\left(\phi_{2 s}+\widehat{\phi}_{s}\right)\right|+\left|q\left(a, \phi_{1 a}+\widehat{\phi}_{a}\right)-q\left(a, \phi_{2 a}+\widehat{\phi}_{a}\right)\right|\right. \\
& +\mid \int_{0}^{a} A T(a-\tau)\left[q\left(\tau, \phi_{1 \tau}+\widehat{\phi}_{\tau}-q\left(\tau, \phi_{2 \tau}+\widehat{\phi}_{\tau}\right)\right] \mathrm{d} \tau \mid\right. \\
& \left.+\left|\int_{0}^{a} T(a-s)\left[f\left(\tau, \phi_{1 \tau}+\widehat{\phi}_{\tau}\right)-f\left(\tau, \phi_{2 \tau}+\widehat{\phi}_{\tau}\right)\right] \mathrm{d} g(\tau)\right|\right](s) \mathrm{d} g(s) \\
\leq & M[g(a)-g(0)]\left[K_{1}\left(1+M M^{\prime}[g(a)-g(0)]\right)+M^{\prime}\left(M L_{p}+H\left\|(-A)^{-\alpha}\right\|\right.\right. \\
& \left.\left.+\frac{H C_{1-\alpha} a^{\alpha}}{\alpha}\right)\right]\left\|\phi_{1}-\phi_{2}\right\| .
\end{aligned}
$$

Therefore, given $\varepsilon>0$, there exists $\delta(\varepsilon)>0$ such that $\left\|\phi_{1}-\phi_{2}\right\|<\delta$ implies $\left|F_{1} \phi_{1}(t)-F_{1} \phi_{2}(t)\right|<\varepsilon$, which proves that operator $F_{1}$ is continuous.
Step III: we shall show that $F_{1}$ maps $B_{l}$ into an equicontinuous family. For $y \in B_{l}, \theta_{1}, \theta_{2} \in J, 0<\theta_{2}<\theta_{1} \leq a$, we have 


$$
\begin{aligned}
\left\|F_{1} y\left(\theta_{1}\right)-F_{1} y\left(\theta_{2}\right)\right\|= & \left\|\int_{0}^{\theta_{1}} T\left(\theta_{1}-s\right) f\left(s, y_{s}+\widehat{\phi}_{s}\right) \mathrm{d} g(s)-\int_{0}^{\theta_{2}} T\left(\theta_{2}-s\right) f\left(s, y_{s}+\widehat{\phi}_{s}\right) \mathrm{d} g(s)\right\| \\
& +\left\|\int_{0}^{\theta_{1}} T\left(\theta_{1}-s\right) B u_{x}(s) \mathrm{d} g(s)-\int_{0}^{\theta_{2}} T\left(\theta_{2}-s\right) B u_{x}(s) \mathrm{d} g(s)\right\| \\
\leq & \left\|\int_{0}^{\theta_{2}}\left[T\left(\theta_{1}-s\right)-T\left(\theta_{2}-s\right)\right] f\left(s, y_{s}+\widehat{\phi}_{s}\right) \mathrm{d} g(s)\right\| \\
& +\left\|\int_{\theta_{2}}^{\theta_{1}} T\left(\theta_{1}-s\right) f\left(s, y_{s}+\widehat{\phi}_{s}\right) \mathrm{d} g(s)\right\| \\
& +\left\|\int_{0}^{\theta_{2}}\left[T\left(\theta_{1}-s\right)-T\left(\theta_{2}-s\right)\right] B u_{x}(s) \mathrm{d} g(s)\right\|+\left\|\int_{\theta_{2}}^{\theta_{1}} T\left(\theta_{1}-s\right) B u_{x}(s) \mathrm{d} g(s)\right\| \\
\leq & \sup _{s \in[0, a]}\left\|T\left(\theta_{1}-s\right)-T\left(\theta_{2}-s\right)\right\|\left\|\int_{0}^{\theta_{2}} f\left(s, y_{s}+\widehat{\phi}_{s}\right) \mathrm{d} g(s)\right\|+M \Phi\left(l^{\prime}\right)\left[H\left(\theta_{1}\right)\right. \\
& \left.-H\left(\theta_{2}\right)\right]+L \sup _{s \in[0, a]}\left\|T\left(\theta_{1}-s\right)-T\left(\theta_{2}-s\right)\right\|\left[g\left(\theta_{2}\right)-g(0)\right]+L M\left[g\left(\theta_{1}\right)-g\left(\theta_{2}\right)\right],
\end{aligned}
$$

where $\quad H(t)=\int_{0}^{t} m(s) \mathrm{d} g(s), \quad L=M^{\prime}\left[\left\|x_{1}\right\|+\right.$ $M\left[\|\phi(0)\|+\left(e l^{\prime}+f\right)+H_{1}\left\|(-A)^{-\alpha}\right\|\left(\left\|x_{0}\right\|+1\right)\right]+$ $H_{1}\left\|(-A)^{-\alpha}\right\|\left(l^{\prime}+1\right)+\left(H_{1} C_{1-\alpha} a^{\alpha}\left(l^{\prime}+1\right) / \alpha\right)+M \Phi\left(l^{\prime}\right)$ $\left.[g(a)-g(0)]^{(1 / q)}\|m\|_{K_{g}^{p}}\right] .\left\|F_{1} y\left(\theta_{1}\right)-F_{1} y\left(\theta_{2}\right)\right\| \longrightarrow 0$, as $\theta_{2} \longrightarrow \theta_{1}$. Hence, $F_{1} B_{l}$ is equicontinuous. Since the case $\theta_{2}<\theta_{1}<0$ or $\theta_{2}<0<\theta_{1}$ is very simple, the proof of the equicontinuities for the two cases is omitted.

Subsequentially, we shall show that $F_{1} B_{l}$ is precompact. Let $0<t \leq a$ be fixed and $\varepsilon$ be a real number satisfying $0<\varepsilon<t$. For $y \in B_{l}$, we define

$$
\begin{aligned}
F_{1}^{\varepsilon} y(t)= & T(\varepsilon) \int_{0}^{t-\varepsilon} T(t-\varepsilon-s) f\left(s, y_{s}+\widehat{\phi}_{s}\right) \mathrm{d} g(s)+T(\varepsilon) \int_{0}^{t-\varepsilon} T(t-\varepsilon-s) B W^{-1}\left[x_{1}-T(a)\right. \\
& \times\left[\phi(0)+p(y+\widehat{\phi})-q\left(0, x_{0}\right)\right]-q\left(a, y_{a}+\widehat{\phi}_{a}\right)-\int_{0}^{a} A T(a-\tau) q\left(\tau, y_{\tau}+\widehat{\phi}_{\tau}\right) \mathrm{d} \tau \\
& \left.-\int_{0}^{a} T(a-\tau) f\left(\tau, y_{\tau}+\widehat{\phi}_{\tau}\right) \mathrm{d} g(\tau)\right] \mathrm{d} g(s) .
\end{aligned}
$$

$T(t)$ is a compact operator, and $Y_{\varepsilon}(t)=\left\{\left(F_{1}^{\varepsilon} y\right)(t): y \in B_{l}\right\}$ is relatively compact in $X$.

For every $\varepsilon, 0<\varepsilon<t$. Moreover, for every $y \in B_{l}$, we have

$$
\begin{aligned}
\left|F_{1} y(t)-F_{1}^{\varepsilon} y(t)\right|= & \int_{t-\varepsilon}^{t} T(t-s) f\left(s, y_{s}+\widehat{\phi}_{s}\right) \mathrm{d} g(s)+\int_{t-\varepsilon}^{t} T(t-s) B W^{-1}\left[x_{1}-T(a)\right. \\
& \left.-q\left(0, x_{0}\right)\right]-q\left(a, y_{a}+\widehat{\phi}_{a}\right)-\int_{0}^{a} A T(a-\tau) q\left(\tau, y_{\tau}+\widehat{\phi}_{\tau}\right) \mathrm{d} \tau \\
& \left.-\int_{0}^{a} T(a-\tau) f\left(\tau, y_{\tau}+\widehat{\phi}_{\tau}\right) \mathrm{d} g(\tau)\right] \mathrm{d} g(s) .
\end{aligned}
$$

$\varepsilon \longrightarrow 0^{+},\left\{\left(F_{1} y\right)(t): Y \in B_{l}\right\}$ is precompact in $X . F_{1} B_{l}$ is uniformly bounded. By the Arzela-Ascoli theorem, it is concluded from the uniform boundedness, equicontinuity, and precompactness of the set $F_{1} B_{l}$ that $\overline{F_{1} B_{l}}$ is compact.

Step IV: $F\left(B_{l}\right)$ is equiregulated on $J$. 


$$
\begin{aligned}
\left\|F x(t)-F x\left(t_{0}^{+}\right)\right\| \leq & \left\|T(t)-T\left(t_{0}^{+}\right)\right\|\left\|\phi(0)+p(x)-q\left(0, x_{0}\right)\right\|+\left\|q\left(t, x_{t}\right)-q\left(t_{0}^{+}, x_{t_{0}^{+}}\right)\right\| \\
& +\left\|\int_{0}^{t_{0}^{+}}(-A)\left[T(t-s)-T\left(t_{0}^{+}-s\right)\right] q\left(s, x_{s}\right) \mathrm{d} s\right\|+\left\|\int_{t_{0}^{+}}^{t}(-A) T(t-s) q\left(s, x_{s}\right) \mathrm{d} s\right\| \\
& +\left\|\int_{0}^{t_{0}^{+}}\left[T(t-s)-T\left(t_{0}^{+}-s\right)\right] B u(s) \mathrm{d} g(s)\right\|+\left\|\int_{t_{0}^{+}}^{t} T(t-s) B u(s) \mathrm{d} g(s)\right\| \\
& +\left\|\int_{0}^{t_{0}^{+}}\left[T(t-s)-T\left(t_{0}^{+}-s\right)\right] f\left(s, x_{s}\right) \mathrm{d} g(s)\right\|+\left\|\int_{t_{0}^{+}}^{t} T(t-s) f\left(s, x_{s}\right) \mathrm{d} g(s)\right\| \\
= & I_{1}+I_{2}+I_{3}+I_{4}+I_{5}+I_{6}+I_{7}+I_{8},
\end{aligned}
$$

where

$$
\begin{aligned}
& I_{1}=\left\|T(t)-T\left(t_{0}^{+}\right)\right\|\left\|\phi(0)+p(x)-q\left(0, x_{0}\right)\right\|, \\
& I_{2}=\left\|q\left(t, x_{t}\right)-q\left(t_{0}^{+}, x_{t_{0}^{+}}\right)\right\|, \\
& I_{3}=\left\|\int_{0}^{t_{0}^{+}}(-A)\left[T(t-s)-T\left(t_{0}^{+}-s\right)\right] q\left(s, x_{s}\right) \mathrm{d} s\right\|, \\
& I_{4}=\left\|\int_{t_{0}^{+}}^{t}(-A) T(t-s) q\left(s, x_{s}\right) \mathrm{d} s\right\|, \\
& I_{5}=\left\|\int_{0}^{t_{0}^{+}}\left[T(t-s)-T\left(t_{0}^{+}-s\right)\right] B u(s) \mathrm{d} g(s)\right\|, \\
& I_{6}=\left\|\int_{t_{0}^{+}}^{t} T(t-s) B u(s) \mathrm{d} g(s)\right\|, \\
& I_{7}=\left\|\int_{0}^{t_{0}^{+}}\left[T(t-s)-T\left(t_{0}^{+}-s\right)\right] f\left(s, x_{s}\right) \mathrm{d} g(s)\right\|, \\
& I_{8}=\left\|\int_{t_{0}^{+}}^{t} T(t-s) f\left(s, x_{s}\right) \mathrm{d} g(s)\right\| .
\end{aligned}
$$

According to Step II of Theroem 1, we can know that $F\left(B_{l}\right)$ is equiregulated on $J$.
Step V: we show that operator $F_{2}$ is a contraction with constant $k$, and we have

$$
\begin{aligned}
\left|F_{2} \phi_{1}(t)-F_{2} \phi_{2}(t)\right| \leq & M\left|p\left(\phi_{1}+\widehat{\phi}\right)-p\left(\phi_{2}+\widehat{\phi}\right)\right|+\left|q\left(t, \phi_{1 t}+\widehat{\phi}_{t}\right)-q\left(t, \phi_{2 t}+\widehat{\phi}_{t}\right)\right| \\
& \quad+\int_{0}^{t} A T(t-s)\left|q\left(s, \phi_{1 s}+\widehat{\phi}_{s}\right)-q\left(s, \phi_{2 s}+\widehat{\phi}_{s}\right)\right| \mathrm{d} s \\
\leq & M L_{p}\left\|\phi_{1}-\phi_{2}\right\|+H\left\|(-A)^{-\alpha}\right\|\left\|\phi_{1}-\phi_{2}\right\|+\frac{a^{\alpha} H C_{1-\alpha}}{\alpha}\left\|\phi_{1}-\phi_{2}\right\| \\
\leq & {\left[M L_{p}+H\left(\left\|(-A)^{-\alpha}\right\|+\frac{a^{\alpha} C_{1-\alpha}}{\alpha}\right)\right]\left\|\phi_{1}-\phi_{2}\right\|:=k\left\|\phi_{1}-\phi_{2}\right\| . }
\end{aligned}
$$

Therefore, all the conditions of Krasnoselskii's fixed point theorem are satisfied, and thus, operator $F_{1}+F_{2}$ has a 
fixed point in $B_{l}$. From this, it follows that operator $F$ has a fixed point and, hence, system (1) is controllable on $J$. This completes the proof.

\section{Example}

Consider the following equation:

$$
\left\{\begin{array}{l}
d_{t}\left[x(t, \theta)-\int_{0}^{\pi} U(\theta, y) x_{t}(s, y) \mathrm{d} y\right]=\frac{\partial^{2}}{\partial \theta^{2}} x(t, \theta) \mathrm{d} t+\left[u(t)+f\left(t, x_{t}(s, \theta)\right)\right] \mathrm{d} g(t), \quad t \in J=[0,1], \theta \in[0, \pi], \\
x(t, 0)=x(t, \pi)=0, \quad t \in[0,1], \\
x(s, \theta)=\phi(s, \theta)+\int_{-(1 / 2)}^{0} h(s) \ln (1+|x(s, \theta)|) \mathrm{d} s, \quad \theta \in[0, \pi], s \in\left[-\frac{1}{2}, 0\right] .
\end{array}\right.
$$

Let $X=L^{2}([0, \pi] ; R)$, we define $A: X \longrightarrow X$ by $A x=$ $\left(\partial^{2} / \partial \theta^{2}\right) x(t, \theta)$ and $D(A)=\{x(\cdot) \in X: x,(\partial x / \partial \theta)$ are absolutely continuous, and $\left(\partial^{2} x / \partial \theta^{2}\right) \in X, x(t, 0)=$ $x(t, \pi)=0\}$. Then, $A$ generates a strongly continuous semigroup $\{T(t), t \geq 0\}$. Furthermore, $A$ has a discrete spectrum, and the eigenvalues are $-n^{2}, n \in N$, with corresponding normalized eigenvectors $x_{n}(z)=(2 / \pi)^{1 / 2} \sin (n z)$. We also use the following properties:

(i) For each $x \in X, T(t) x$ : $=\sum_{n=1}^{\infty} e^{-n^{2} t}\left\langle x, x^{n}\right\rangle x^{n}$. In particular, $T(\cdot)$ is a uniformly stable semigroup and $\|T(t)\|_{L^{2}[0, \pi]} \leq e^{-t} \leq 1$.

(ii) For each $x \in X,(-A)^{-(1 / 2)} x=\sum_{n=1}^{\infty}(1 / n)\left\langle x, x^{n}\right\rangle x^{n}$. In particular, $\left\|(-A)^{1 / 2}\right\|_{L^{2}[0, \pi]}=1$.

(iii) The operator $(-A)^{(1 / 2)}$ is given by $(-A)^{(1 / 2)} x=$ $\sum_{n=1}^{\infty} n\left\langle x, x^{n}\right\rangle x^{n}$, on the space $D\left((-A)^{-(1 / 2)}\right)=$ $\left\{x(\cdot) \in X, \sum_{n=1}^{\infty} n\left\langle x, x^{n}\right\rangle x^{n} \in X\right\}$.

Obviously, Lemma 5 and (H1) are satisfied. Let $\left(U_{h} x\right)(\theta)=\int_{0}^{\pi} U(\theta, y) x(y) \mathrm{d} y, \quad$ for $\quad x \in X, \theta \in$ $[0, \pi]$. Then, function $f:(0,1] \times G([-(1 / 2)$,
$0] ; X) \longrightarrow X$ is continuous. Let $F\left(t, x_{t}(\theta)\right)=$ $f\left(t, x_{t}\right) \quad(\theta)=a \sin \left(x_{t}(\theta)\right), t \in[0,1], \theta \in[0, \pi]$, where $a$ is a constant. We define

Let $m(t)=a, \Phi(l)=l$, and we can see that $\varphi=1$.

$$
\begin{aligned}
\left\|f\left(t, x_{t}\right)(\theta)\right\| & =\left(\int_{0}^{\pi} a^{2} \sin ^{2}\left(x_{t}(\theta)\right) d \theta\right)^{(1 / 2)} \\
& \leq\left(\int_{0}^{\pi} a^{2}\left(x_{t}(\theta)\right)^{2} d \theta\right)^{(1 / 2)} \\
& =a\left\|x_{t}\right\| \\
\left\|f\left(t, x_{t}^{n}\right)-f\left(t, x_{t}\right)\right\| & =\left(\int_{0}^{\pi} a^{2}\left|\sin \left(x_{t}^{n}(\theta)\right)-\sin \left(x_{t}(\theta)\right)\right|^{2} \mathrm{~d} \theta\right)^{(1 / 2)} \\
& \leq a\left(\int_{0}^{\pi}\left|\left(x_{t}^{n}(\theta)\right)-\left(x_{t}(\theta)\right)\right|^{2} \mathrm{~d} \theta\right)^{(1 / 2)} \\
& \leq a\left|x_{t}^{n}-x_{t}\right| .
\end{aligned}
$$

Therefore, (H3) is satisfied. $p: G([-(1 / 2), 1] ; X) \longrightarrow X$, and $p(\phi)(\theta)=\int_{-(1 / 2)}^{0} h(s)$ $\ln (1+|\phi(s)(\theta)|) \mathrm{d} s, \quad \phi \in G([-(1 / 2), 1] ; X), \quad \phi(s)(\theta)=$ $x(s, \theta)$. Supposing that $h \in L([-(1 / 2), 0] ; R)$ and letting $\left\{\phi_{n}, n \in N\right\}$ be a convergence sequence on $G([-(1 / 2), 1] ; X)$, 


$$
\begin{aligned}
\left\|p\left(\phi_{n}\right)-p(\phi)\right\|_{X}^{2} & =\int_{0}^{\pi}\left|p\left(\phi_{n}\right)(\theta)-p(\phi)(\theta)\right|^{2} \mathrm{~d} \theta, \\
& =\int_{0}^{\pi}\left(\int_{-(1 / 2)}^{0} h(s)\left(\ln \left(1+\phi_{n}(s)(\theta)\right)-\ln (1+\phi(s)(\theta))\right) \mathrm{d} s\right)^{2} \mathrm{~d} \theta \\
& \leq \int_{-(1 / 2)}^{0}|h(s)|^{2} \mathrm{~d} s \int_{-(1 / 2)}^{0} \int_{0}^{\pi}\left|\phi_{n}(s)(\theta)-\phi(s)(\theta)\right|^{2} \mathrm{~d} \theta \mathrm{d} s \longrightarrow 0,
\end{aligned}
$$

as $n \longrightarrow \infty$. Thus, $p$ is a continuous operator. Using the same method, we can obtain that, for any $\phi \in G([-(1 / 2), 1] ; X),\|p(\phi)(\theta+\eta)-p(\phi)(\theta)\|_{X} \longrightarrow$ 0 , as $\quad \eta \longrightarrow 0$. Moreover, $\|p(\phi)\|_{X} \leq$ $\left(\int_{-(1 / 2)}^{0}|h(s)|^{2} \mathrm{~d} s\right)^{(1 / 2)}\|\phi\|_{\infty}=e\|\phi\|_{\infty}$. Therefore, (H4) is satisfied.

(iv) The function $U(\theta, y), \theta, y \in[0, \pi]$ is measurable and $\int_{0}^{\pi} \int_{0}^{\pi} U^{2}(\theta, y) \mathrm{d} y \mathrm{~d} \theta<\infty$.

(v) The function $\partial_{\theta} U(\theta, y)$ is measurable, and $U(0, y)=U(\pi, y)=0$; let

$$
\bar{H}=\left(\int_{0}^{\pi} \int_{0}^{\pi}\left(\partial_{\theta} U(\theta, y)\right)^{2} \mathrm{~d} y \mathrm{~d} \theta\right)^{(1 / 2)}<\infty .
$$

From (iv) it is clear that $U_{h}$ is a bounded linear operator on $X$. Furthermore, $U_{h}(x) \in D\left((-A)^{(1 / 2)}\right)$, and $\left\|\left((-A)^{-(1 / 2)}\right) U_{h}\right\|_{L^{2}[0, \pi]}<\infty$.

In fact, from the definition of $U_{h}$ and (v), it follows that

$$
\begin{aligned}
\left\langle U_{h}(x), x^{n}\right\rangle & =\int_{0}^{\pi} x^{n}(\theta)\left(\int_{0}^{\pi} U(\theta, y) x(y) \mathrm{d} y\right) \mathrm{d} \theta \\
& =\frac{1}{n}\left(\frac{2}{\pi}\right)^{(1 / 2)}\langle\bar{U}(x), \cos (n \theta)\rangle
\end{aligned}
$$

where $\bar{U}$ is defined by $\bar{U}(x)(\theta)=\int_{0}^{\pi} \partial_{\theta} U(\theta, y) x(y) \mathrm{d} y$.

From (v) we know that $\bar{U}: X \stackrel{X}{\longrightarrow}$ is a bounded linear operator with $\|\bar{U}\|_{L^{2}[0, \pi]} \leq \bar{H}$. Hence, we can write $\left\|(-A)^{(1 / 2)} U_{h}(x)\right\|_{L^{2}[0, \pi]}=\|\bar{U}(x)\|_{L^{2}[0, \pi]}$, which implies that (H2) holds. Hence, according to Theorem 1, system (38) has a mild solution provided that the condition of Theorem 1 holds. Moreover, we can impose suitable hypotheses to verify the assumptions stated in Theorem 2. Thus, system (38) is controllable on $[0,1]$.

\section{Conclusions}

In this paper, the issue on approximate controllability of neutral measure evolution equations has been addressed, which can model a large class of hybrid systems without any restriction on their Zeno behavior. Firstly, by adopting Schauder's fixed point theorem, the existence results of mild solutions for this type of measure control system corresponding to some control function are obtained. Then, the approximate controllability results are provided. Finally, we also use an example to illustrate the main result. Furthermore, we will investigate measure functional evolution equations of Sobolev type in the next work.

\section{Data Availability}

There are no underlying data in the results.

\section{Conflicts of Interest}

The authors declare that they have no conflicts of interest.

\section{Authors' Contributions}

All authors read and approved the final manuscript.

\section{Acknowledgments}

This work was supported by the Outstanding Young Science and technology Training program of Xinjiang (2019Q022), National Natural Science Foundation of China (11961069 and 11861068), Natural Science Foundation of Xinjiang (2019D01A71 and 2018D01A27), Scientific Research Programs of Colleges in Xinjiang (XJEDU2018Y033), and Xinjiang Normal Uniersity Graduate Research Innovation Project (XSY202002002).

\section{References}

[1] M. Benchohra, S. Litimein, J. J. Nieto et al., "Semilinear fractional differential equations with infinite delay and noninstantaneous impulses," Journal of Fixed Point Theory and Applications, vol. 21, no. 1, pp. 1-16, 2019.

[2] S. Liu, A. Debbouche, and J. Wang, "ILC method for solving approximate controllability of fractional differential equations with noninstantaneous impulses," Journal of Computational and Applied Mathematics, vol. 339, no. 2018, pp. 343-355, 2018.

[3] M. Muslim, A. Kumar, M. Feckan et al., "Existence, uniqueness and stability of solutions to second order nonlinear differential equations with non-instantaneous impulses," Journal of King Saud University - Science, vol. 30, no. 2, pp. 204-213, 2016.

[4] Y. Cao and J. Sun, "Existence of solutions for semilinear measure driven equations," Journal of Mathematical Analysis and Applications, vol. 425, no. 2, pp. 621-631, 2015.

[5] Y. Cao and J. Sun, "Measures of noncompactness in spaces of regulated functions with application to semilinear measure driven equations," Boundary Value Problems, vol. 2016, no. 1, pp. 1-17, 2016.

[6] Y. Cao and J. Sun, "Practical stability of nonlinear measure differential equations," Nonlinear Analysis: Hybrid Systems, vol. 30, pp. 163-170, 2018.

[7] Y. Cao and J. Sun, "Approximate controllability of semilinear measure driven systems," Mathematische Nachrichten, vol. 291, no. 13, pp. 1979-1988, 2018. 
[8] Y. Cao and J. Sun, "Controllability of measure driven evolution systems with nonlocal conditions," Applied Mathematics and Computation, vol. 299, pp. 119-126, 2017.

[9] Y. Cao and J. Sun, "On existence of nonlinear measure driven equations involving non-absolutely convergent integrals," Nonlinear Analysis: Hybrid Systems, vol. 20, pp. 72-81, 2016.

[10] L. P. Rodrigo, M. A. Ignacio, and R. L. Jorge, "Solvability of non-semicontinuous systems of Stieltjes differential inclusions and equations," Advances in Difference Equations, 2020.

[11] M. Cichon and B. Satco, "Measure differential inclusions between continuous and discrete," Advances in Difference Equations, vol. 2014, no. 1, pp. 1-18, 2014.

[12] P. C. Das and R. R. Sharma, "On optimal controls for measure delay-differential equations," SIAM Journal on Control, vol. 9, no. 1, pp. 43-61, 1971.

[13] P. C. Das and R. R. Sharma, "Existence and stability of measure differential equations," Czechoslovak Mathematical Journal, vol. 22, no. 1, pp. 145-158, 1972.

[14] M. Federson, J. G. Mesquita, and A. Slavik, "Measure functional differential equations and functional dynamic equations on time scales," Journal of Differential Equations, vol. 252, no. 6, pp. 3816-3847, 2012.

[15] M. Federson, R. Grau, and J. G. Mesquita, "Boundedness of solutions of measure differential equations and dynamic equations on time scales," Journal of Differential Equations, vol. 263, no. 1, pp. 26-56, 2017.

[16] G. N. Silva and R. B. Vinter, "Measure driven differential inclusions," Journal of Mathematical Analysis and Applications, vol. 202, no. 3, pp. 727-746, 1996.

[17] A. Slavík, "Well-posedness results for abstract generalized differential equations and measure functional differential equations," Journal of Differential Equations, vol. 259, no. 2, pp. 666-707, 2015.

[18] A. Slavík, "Measure functional differential equations with infinite delay," Nonlinear Analysis: Theory, Methods \& Applications, vol. 79, pp. 140-155, 2013.

[19] M. Federson, R. Grau, and J. G. Mesquita, "Lyapunov stability for measure differential equations and dynamic equations on time scales," Journal of Differential Equations, vol. 267, no. 7, pp. 4192-4223, 2019.

[20] C. A. Gallegos, H. R. Henríquez, J. G. Mesquita et al., "Growth of solutions for measure differential equations and dynamic equations on time scales," Journal of Mathematical Analysis and Applications, vol. 479, no. 1, pp. 941-962, 2019.

[21] H. Gu and Y. Sun, "Nonlocal controllability of fractional measure evolution equation," Journal of Inequalities and Applications, 2020.

[22] Y. Shi, H. Gu, X. Yan, and C. Zheng, "Semilinear functional measure drive equation with nonlocal conditions," Journal of Xinjiang Normal University (Natural Sciences Edition), vol. 36, no. 2, pp. 64-68, 2017.

[23] Y. K. Chang, "Controllability of impulsive functional differential systems with infinite delay in Banach spaces," Chaos, Solitons \& Fractals, vol. 33, no. 5, pp. 1601-1609, 2007.

[24] J. P. Dauer and N. I. Mahmudov, "Integral inequalities and mild solutions of semilinear neutral evolution equations," Journal of Mathematical Analysis and Applications, vol. 300, no. 1, pp. 189-202, 2004.

[25] V. Kavitha and M. Mallika Arjunan, "Controllability of nondensely defined impulsive neutral functional differential systems with infinite delay in Banach spaces," Nonlinear Analysis: Hybrid Systems, vol. 4, no. 3, pp. 441-450, 2010.

[26] A. Kumar, R. Vats, and A. Kumar, "Approximate controllability of second-order non-autonomous system with finite delay," Journal of Dynamical and Control Systems, vol. 26, no. 1, pp. 611-627, 2020.

[27] M. Muslim, A. Kumar, and R. Sakthivel, "Exact and trajectory controllability of second-order evolution systems with impulses and deviated arguments," Mathematical Methods in the Applied Sciences, vol. 41, no. 11, pp. 4259-4272, 2018.

[28] H. Qin, J. Liu, and X. Zuo, "Controllability problem for fractional integrodifferential evolution systems of mixed type with the measure of noncompactness," Journal of Inequalities and Applications, vol. 2014, no. 1, pp. 292-307, 2014.

[29] S. Rathinasamy and R. Yong, "Approximate controllability of fractional differential equations with state-dependent delay," Results in Mathematics, vol. 63, no. 3-4, pp. 949-963, 2013.

[30] Z. Tai and X. Wang, "Controllability of fractional-order impulsive neutral functional infinite delay integrodifferential systems in Banach spaces," Applied Mathematics Letters, vol. 22 , no. 11, pp. 1760-1765, 2009.

[31] Z. Tai, "Controllability of fractional impulsive neutral integrodifferential systems with a nonlocal Cauchy condition in Banach spaces," Applied Mathematics Letters, vol. 24, no. 12, pp. 2158-2161, 2011.

[32] A. Pazy, Semigroups of Linear Operators and Applications to Partial Differential Equations, Springer-Verlag, Berlin, Germany, 1983.

[33] Y. Zhou and F. Jiao, "Nonlocal Cauchy problem for fractional evolution equations," Nonlinear Analysis: Real World Applications, vol. 11, no. 5, pp. 4465-4475, 2010. 